\title{
COresponsabilité budgétaire et révision de la LAMal: Managed care / Soins intégrés
}

\author{
medswiss.net, l'association suisse des réseaux de médecins, a été le premier rédacteur \\ à utiliser et à marquer de son empreinte la notion de «coresponsabilité budgétaire»; \\ elle en présente la définition suivante.
}

Le projet de loi relatif aux soins intégrés (managed care) vise à accorder aux assurés une quote-part réduite lorsqu'ils optent pour un modèle d'assurancemaladie qui prévoit la coresponsabilité budgétaire des fournisseurs de prestations.

Aujourd'hui établie dans l'usage, la notion de «coresponsabilité budgétaire» a été créée en 2005 par un groupe de travail de medswiss.net, l'association suisse des réseaux de médecins, dans le cadre de la définition du réseau de médecins «Vision 08». En choisissant cette expression, medswiss.net a tenu à indiquer qu'il n'a jamais été question de déplacer, dans les conventions de managed care, la responsabilité économique exclusivement sur les réseaux de médecins, mais que cette responsabilité est assumée par les deux partenaires contractuels, assureur et réseau de médecins.

La présente définition de medswiss.net prend en compte les expériences acquises depuis 15 ans en Suisse avec le managed care.

Les éléments centraux de la responsabilité économique sont l'ensemble (assurés et fournisseurs de prestations), le budget et la participation au bénéfice / au déficit.

\section{Définition «coresponsabilité budgétaire»}

- La coresponsabilité budgétaire est une responsabilité économique qui a été négociée par les partenaires cosignataires d'une convention de managed care en vue d'assumer ensemble les coûts de l'assurance obligatoire des soins d'un ensemble d'assurés selon un modèle de managed care.

- Les partenaires contractuels sont d'une part les assureurs et d'autre part un ensemble de fournisseurs de prestations.

- Les partenaires contractuels négocient une convention sur les prestations (convention de managed care / convention de soins intégrés).

- La coresponsabilité budgétaire est une part intégrante négociable de la convention; elle comprend le budget et la participation au bénéfice / au déficit.

Dr Jörg Fritschi Kleinbuholz 3 CH-6012 Obernau

j.fritschi@hin.ch

- Le budget établi pour le groupe d'assurés doit être calculé en fonction des risques. Les indica-

\section{Groupe d'assurés et de fournisseurs de prestations}

Le managed care, c'est-à-dire les soins intégrés, ne peut être mis en œuvre de manière responsable que par l'intermédiaire d'un ensemble d'assurés et de fournisseurs de prestations médicales. Les fournisseurs de prestations s'organisent à cet effet sous une forme de société appropriée (par ex. association, société anonyme).

\section{Budget}

Le budget est un projet négociable sur les objectifs à atteindre en termes de coûts pour la gestion d'un ensemble d'assurés. Les éléments de la négociation portent sur le montant du budget, la manière de le calculer, son ampleur et l'activité concernée.

\section{Méthodes de calcul}

Les méthodes de calcul habituelles sont les suivantes:

- Comparaison avec un groupe d'assurés conventionnels (système de calcul parallèle);

- Comparaison aux coûts de l'exercice précédent avec prise en compte du renchérissement (système de calcul par rapport à l'exercice précédent);

teurs de risque peuvent être fixés contractuellement (données relatives aux personnes, indicateurs de morbidité adéquats ${ }^{1}$, paramètres relevant du domaine de l'assurance ${ }^{2}$ ).

- Les modalités de la participation au bénéfice / au déficit sont fixées par les partenaires contractuels.

- Le budget et la coresponsabilité budgétaire s'appliquent uniquement à des collectifs. Le budget concerne le collectif des assurés tandis que la coresponsabilité budgétaire est assumée par le collectif des fournisseurs de prestations.

(Elaborée par les réseaux de médecins regroupés dans l'association suisse) par ex. préhospitalisations, pharmaceutical cost groups

2 montant de la franchise de l'assuré, économies visées, seuil minimal à la charge de l'assurance pour couvrir les cas avec des coûts élevés, etc. 
- Capitation ou forfait par tête en tant que méthode de calcul prospective sur la base des données individuelles des assurés en fonction de leurs maladies. Les personnes en bonne santé sont également incluses dans le budget, ce qui incite particulièrement à la prévention.

Remarque: la capitation est seulement un instrument du calcul et n'entraîne pas automatiquement une coresponsabilité économique.

\section{Ampleur du budget}

Le budget peut comprendre toute la chaîne thérapeutique ou une partie seulement de celle-ci. Il faut donner la préférence aux conventions englobant toute la chaîne thérapeutique car on évite ainsi les incitatifs à déplacer les risques dans d'autres secteurs de la santé.

Les réseaux de médecins sont prêts à inclure aussi des personnes atteintes de maladies chroniques dans les soins intégrés, à la condition de calculer le budget en fonction de la morbidité.

\section{Activité}

Sans participation au bénéfice / au déficit, un budget reste informel et correspond au «simple» contrat de médecin de famille servant de modèle d'accès aux soins intégrés pour les jeunes réseaux de médecins.

Seule la participation au bénéfice ou au déficit (participation au risque*) rend le budget actif et signifie coresponsabilité économique.

\section{Participation au bénéfice/déficit}

La participation au bénéfice ou au déficit est l'élément central de la coresponsabilité économique. Elle est également négociable. En règle générale, le montant budgétisé n'est pas versé au réseau de médecins; il reste donc virtuel. Ce montant n'est pas le paiement d'une prestation.

Le montant inscrit au budget et les coûts effectifs font l'objet d'une comparaison, ce qui permet de dégager un bénéfice ou un déficit. La convention de prestations entre l'assureur et le réseau de médecins détermine la forme et l'importance de la participation. On prévoit habituellement une participation sous forme de bonus/malus ou une participation en pour cent. Pour la participation au bénéfice, ou à une perte, c'est toujours le réseau qui est responsable, jamais ses membres isolés.

D'autres mesures de sécurité peuvent être prévues: limitation, réserves, réassurances, assurance des risques élevés. Une responsabilité économique complète est donnée lorsque le risque est entièrement assumé par le réseau de médecins dans le cadre d'un budget global. Le réseau de médecins reprend alors le rôle de l'assureur.
L'association suisse des réseaux de médecins s'est toujours prononcée en faveur d'une séparation des fournisseurs de prestations et des répondants des coûts. En effet, le domaine de l'assurance et celui de la médecine exigent chacun des compétences propres. Si ces deux domaines étaient regroupés, les soins de santé seraient pilotés sur le plan financier par un budget global.

La sécurité des soins est-elle aussi garantie dans le cadre de la coresponsabilité budgétaire? Clairement oui!

La coresponsabilité budgétaire garantit même la sécurité des soins. La solution s'appelle équilibre des soins! Dans l'assurance-maladie conventionnelle, les prestations sont facturées individuellement, ce qui peut mener à une surabondance de soins qui n'est pas toujours sans danger.

D'un autre côté, un budget à lui seul tendrait vers un sous-approvisionnement en soins. Dans un réseau de médecins avec coresponsabilité budgétaire, les médecins établissent leurs factures selon le tarif des prestations individuelles. En même temps, ils sont tenus de respecter le budget virtuel du réseau. Cette manière de procéder permet d'équilibrer les deux tendances, celle de la surabondance et celle du sousapprovisionnement. Cet équilibre garantit la sécurité des soins.

\section{Imposer par la loi?}

Comment le managed care doit-il / peut-il être imposé par la loi?

Compris comme un système de soins pilotés et intégrés, le managed care est davantage qu'un produit. Il repose sur la disponibilité de tous les participants à travailler ensemble. Cette collaboration présuppose la confiance. Des prescriptions rigides peuvent empêcher la conclusion de conventions managed care. En revanche, il est nécessaire de fixer de bonnes conditions cadres légales susceptibles de promouvoir le managed care.

Association suisse des réseaux de médecins
medswiss.net
L'association comprend 47 organisations de
médecins de famille et réseaux de médecins avec
quelque 2000 médecins s'occupant de 600000 as-
surés selon un modèle managed care. En tant
qu'ensembles tenus de respecter des normes
contraignantes, les réseaux de médecins sont
en mesure de répondre aux critères «efficacité,
adéquation, économicité et qualité» exigés par la
LAMal et de les appliquer de même que d'offrir
avec les assureurs intéressés des soins de santé
axés sur les besoins.

